\title{
Ocular Pharmacokinetic/Pharmacodynamic Modeling for Timolol in Rabbits Using a Telemetry System
}

\author{
Koji Sakanaka, ${ }^{*, a, b}$ Kouichi Kawazu, ${ }^{a}$ Masahide Tomonari, ${ }^{b}$ Takashi Kitahara, ${ }^{b}$ \\ Mikiro Nakashima, ${ }^{c}$ Koyo Nishida, ${ }^{c}$ Junzo Nakamura, ${ }^{c}$ Hitoshi Sasaki, ${ }^{b}$ and Shun Higuchi ${ }^{d}$ \\ ${ }^{a}$ Santen Pharmaceutical Co., Ltd., Nara Research and Development Center; 8916-16 Takayama-cho, Ikoma 630-0101, \\ Japan: ${ }^{b}$ Department of Hospital Pharmacy, Nagasaki University School of Medicine; 1-7-1 Sakamoto, Nagasaki \\ 852-8501, Japan: ' Graduate School of Biomedical Sciences, Nagasaki University; 1-14 Bunkyo-machi, Nagasaki \\ 852-8521, Japan: and ${ }^{d}$ Graduate School of Pharmaceutical Sciences, Kyushu University; 3-1-1 Maidashi, Higashi-ku, \\ Fukuoka 812-8582, Japan.
}

Received November 19, 2007; accepted February 25, 2008; published online February 26, 2008

\begin{abstract}
We have established an ocular pharmacokinetic/pharmacodynamic (PK/PD) model for a $\beta$-adrenergic antagonist, timolol, after instillation into rabbits. Timolol concentrations were determined by HPLC in the tear fluid, aqueous humor, cornea, and iris-ciliary body after instillation or ocular injection into the anterior chamber of the eye in rabbits. In addition, intraocular pressure (IOP) measurement was performed after instillation of timolol by a telemetry system, which was able to obtain detailed IOP data automatically. The PK/PD parameters were estimated by fitting the concentration-time profiles and the ocular hypotensive effect-time profiles using MULTI (RUNGE) program. The PK model consisted of six compartments and the PD model included aqueous humor dynamics based on an action mechanism of timolol, which causes lowering of IOP by suppressing aqueous humor production. The PK/PD model described well the concentration-time profiles and the ocular hypotensive effect-time profiles after instillation of timolol. This study is the first trial to develop an ocular PK/PD model for timolol after instillation. This model can predict both the drug concentrations in various ocular tissues and the ocular hypotensive effect after instillation of timolol.
\end{abstract}

Key words pharmacokinetic; pharmacodynamic; eye; timolol; telemetry system

Intraocular pressure (IOP) is mainly determined by the dynamics of the aqueous humor, which is the combination of aqueous humor formation in the ciliary body and its drainage through the uveoscleral and the trabecular meshwork routes. In the current design of glaucoma therapies, reduction in IOP is a major target by instillation of ocular hypotensive drugs. The action mechanisms of these drugs are well known: $\beta$ adrenergic antagonists, carbonic anhydrase inhibitors, and $\alpha_{2}$-adrenergic agonists suppress aqueous humor formation, while prostaglandin FP receptor agonists, prostamides, and $\alpha_{1}$-adrenergic antagonists promote aqueous humor outflow through the uveoscleral route. ${ }^{1,2)}$ However, there have been no quantitative mathematical models to correlate the alteration in the aqueous humor dynamics to the exposure of drugs.

Pharmacodynamics is a quantitative study of a functional relationship between drug concentration and its effect. In order to comprehend the pharmacological and pharmacokinetic properties of a drug, pharmacokinetic/pharmacodynamic (PK/PD) modeling has emerged as a major tool that allows us to predict both drug concentration and pharmacological effect, quantitatively. In the ocular hypotensive drugs, the correlation between the effect and drug concentration in the ocular tissues has been reported to optimize the ophthalmic formulation for ocular drug delivery improvement. ${ }^{3,4)}$ These empirical approaches could not predict ocular hypotensive effects after instillation of the drugs quantitatively because no appropriate ocular PK/PD model has been devised.

Previously, we have succeeded in developing an ocular PK/PD model including aqueous humor dynamics after instillation of an $\alpha_{1}$-adrenergic antagonist, bunazosin. ${ }^{5)}$ This model predicted not only the drug concentration in the ocular tissues but also the intensity and duration of its ocular hypotensive effect after instillation of bunazosin quantitatively.

Timolol, a representative $\beta$-adrenergic antagonist, is one of the most frequently prescribed drugs for glaucoma. The action mechanism of timolol in suppressing aqueous humor formation is different from that of bunazosin, which promotes aqueous humor outflow through the uveoscleral route. There is no ocular PK/PD model for timolol though several ocular PK models have been reported. ${ }^{3,6}$

In the present study, we developed a new ocular PK/PD model for timolol including aqueous humor dynamics based on an action mechanism for timolol in rabbits. We also applied a telemetry system to measure continuous ocular hypotensive effects for PK/PD analysis. This system is able to automatically obtain detailed IOP data, which is preferable for PK/PD analysis, after instillation of drugs into rabbits.

\section{MATERIALS AND METHODS}

Animals Male Japanese White rabbits $(2.0-3.6 \mathrm{~kg})$ were housed individually in cages in an air-conditioned room and maintained on a standard laboratory diet (ORC4 or LRC4, Oriental Yeast Co., Ltd., Tokyo, Japan). The rabbits had free access to water and were maintained in a 12-h lightdark cycle (light on was defined as 7:00 a.m., and light off occurred at 7:00 p.m.). All experiments in the present study conformed to the "Principles of Laboratory Animal Care" (NIH publication \# 85-23, received 1985).

Materials Timolol maleate was purchased from Wako Pure Chemical Ind., Ltd. (Osaka, Japan). All other chemicals used were reagent grade. Drug solutions were prepared with pH 7.4 phosphate-buffered saline (PBS).

Drug Disposition after Instillation Ocular instillation 
and tissue collection were performed as in a previous report. ${ }^{5)}$ Briefly, $25 \mu \mathrm{l}$ of drug solution (timolol: $100 \mathrm{~mm}$ ) was carefully instilled with a micropipette in the middle of the lower conjunctival sac of unanaesthetized rabbits placed in restraint boxes. Tear fluid $(0.5 \mu \mathrm{l})$ was collected using a glass capillary at the designated times and diluted with $0.5 \mathrm{ml}$ PBS. Under the same condition, the rabbits were sacrificed by an overdose of sodium pentobarbital at the designated time after drug instillation. After thoroughly rinsing the corneal and conjunctival surfaces with $0.9 \% \mathrm{NaCl}$, the aqueous humor, cornea, and iris-ciliary body were collected. The diluted tear fluid, aqueous humor, and other ocular tissues were stored at $-20^{\circ} \mathrm{C}$ until drug determination.

Drug Disposition after Ocular Injection The ocular injection method was performed as in a previous report.5) Briefly, after instillation of $0.4 \%$ oxybuprocaine hydrochloride to unanaesthetized rabbits, $1 \mu \mathrm{l}$ of drug solution (timolol: $5 \mathrm{~mm}$ ) was carefully injected into the anterior chamber using a microsyringe with a 30-gauge needle to prevent aqueous humor leakage. The protocol for collecting the aqueous humor and other tissues was described above.

Drug Determination Drug determination was performed using a method slightly modified from a previous report. ${ }^{7)}$ To the diluted tear fluid, $100 \mu \mathrm{l}$ methanol including methyl- $p$-hydroxybenzoate $(500 \mu \mathrm{g} / \mathrm{ml})$ as an internal standard was added. To a $50 \mu \mathrm{l}$ aliquot of aqueous humor, $50 \mu \mathrm{l}$ $\mathrm{HCl}(0.1 \mathrm{M})$ and $100 \mu \mathrm{l}$ methanol including propranolol $(50 \mu \mathrm{M})$ as an internal standard were added. After mixing, each mixture was centrifuged at $2000 \times \boldsymbol{g}$ for $10 \mathrm{~min}$, and $50 \mu \mathrm{l}$ of supernatant was injected into an HPLC system for analysis.

The cornea and iris-ciliary body were homogenized using a glass homogenizer with a mixture of $1 \mathrm{ml}$ water and $100 \mu \mathrm{l}$ methanol including propranolol $(50 \mu \mathrm{M})$. The homogenized mixture was centrifuged at $2000 \times \boldsymbol{g}$ for $10 \mathrm{~min}$, and an aliquot of supernatant was mixed with $200 \mu \mathrm{l}$ perchloric acid $(0.6 \%)$ and centrifuged at $2000 \times \boldsymbol{g}$ for $10 \mathrm{~min}$. To the deproteinized supernatant, $2 \mathrm{ml}$ diethyl ether and $0.9 \mathrm{ml}$ ammonium acetate $(1 \mathrm{M}, \mathrm{pH} 9)$ were added, and the mixture was shaken for $5 \mathrm{~min}$. After centrifugation at $2000 \times \boldsymbol{g}$ for $5 \mathrm{~min}$, the upper organic layer was retained, and $200 \mu \mathrm{l} \mathrm{HCl}(0.2 \mathrm{M})$ was added to the organic layer. The mixture was shaken for $5 \mathrm{~min}$ and centrifuged at $2000 \times \boldsymbol{g}$ for $10 \mathrm{~min}$, the organic layer was discarded and $50 \mu 1$ of the aqueous phase was injected into the HPLC system for analysis.

The HPLC system (LC-10AD, Shimadzu Co., Ltd., Kyoto, Japan) was used in a reversed-phase mode for the assay. The stationary phase used was a Cosmosil 5C18-MS packed column $(150 \mathrm{~mm}$ length $\times 4.6 \mathrm{~mm}$ i.d., Nacalai Tesque Inc., Kyoto, Japan). A mixture of methanol and $3 \mathrm{~mm}$ diethylamine $(3: 7 \mathrm{v} / \mathrm{v})$ was used as the mobile phase with a flow rate of $1.0 \mathrm{ml} / \mathrm{min}$. Retention of the drug was monitored with a UV spectrophotometric detector at $290 \mathrm{~nm}$ (SPD-10A, Shimadzu Co., Ltd., Kyoto, Japan).

Implantation of a Telemetry Transmitter Implantation of a telemetry transmitter was performed as in previous reports. ${ }^{8,9)}$ Briefly, rabbits were anesthetized with an intramuscular injection of $40 \mathrm{mg} / \mathrm{kg}$ ketamine plus $4 \mathrm{mg} / \mathrm{kg}$ xylazine and also received indomethacin $(10 \mathrm{mg} / \mathrm{kg}$ intraperitoneally). In addition, $50 \mu \mathrm{l}$ of $0.1 \%$ diclofenac sodium solution and $0.4 \%$ oxybuprocaine hydrochloride solution were instilled into the right eye. The telemetry transmitter (Model TA11PA-C40; Data Science International, St. Paul, MN, U.S.A.) was inserted into a subcutaneous pocket prepared in the cheek, and its pressure catheter was tunneled subcutaneously to an exit site near the superior conjunctival sac. The sensor catheter was inserted into the midvitreous through a small hole in the sclera, and was tied with nylon sutures. After the surgery, $0.3 \%$ ofloxacin solution and $0.1 \%$ sodium diclofenac solution were instilled into the right eye three times per day for approximately $7 \mathrm{~d}$.

Data Management of IOP by a Telemetry System Data management of IOP was performed using a method slightly modified from previous reports. ${ }^{8,9)}$ The implanted telemetry transmitter sent IOP information to a receiver (RPC-1; Data Science International), and the information was transferred to a computer-based data-acquisition system through the data-exchange matrix (DEM; Data Science International). Each rabbit's IOP information was measured continuously at $100 \mathrm{~Hz}$ for $15 \mathrm{~s}$ in cycle runs of $2.5 \mathrm{~min}$ and the average value was saved. IOP values were determined by the 15 min-average of saved values using the interval-averaging routine of Dataquest Analysis Software (Dataquest ART ${ }^{\mathrm{TM}}$ version $2.3 \mathrm{G}$, Data Science International). The rabbits were used in this study after confirming their IOP values which displayed a stable circadian rhythm across the dark and light phase, and which correlated with the value measured by a pneumatonometer.

IOP Measurement after Instillation Rabbits implanted with the telemetry transmitter and trained enough to be handled were placed in restraint boxes. Twenty-five microliters of each drug solution (timolol: $0.2 \mathrm{~mm}, 1 \mathrm{~mm}, 5 \mathrm{~mm}, 15.8 \mathrm{~mm}$, or $100 \mathrm{~mm}$ ) or vehicle was carefully instilled into the middle of the lower conjunctival sac of the right eye of the unanesthetized rabbits at 11:00 p.m. under reduced red-colored lighting in the dark. The IOP value was obtained every $15 \mathrm{~min}$ until $7 \mathrm{~h}$ after instillation. IOP measurement was performed by the telemetry system as described above. Implanted rabbits were used repeatedly after cessation of each drug.

Ocular Pharmacokinetic Model for Timolol The concentration-time profiles for timolol in the tear fluid (Fig. 1) were analyzed by a two-compartment model. The concentration $\left(C_{\mathrm{T}}\right)$ at time $t$ is expressed as follows:

$C_{\mathrm{T}}=A \cdot e^{-\alpha \cdot t}+B \cdot e^{-\beta \cdot t}$

Hybrid parameters, $A, B, \alpha$, and $\beta$ are defined as

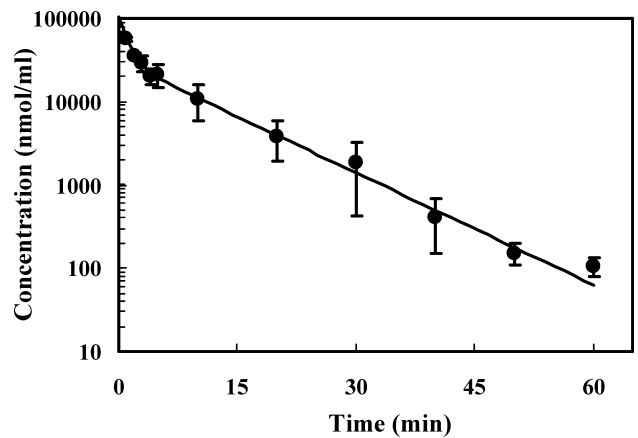

Fig. 1. Concentration of Timolol in Tear Fluid after Instillation

- Experimental data and ( - ) fitted curve. Each point represents the mean \pm S.E. of 6 experiments. 

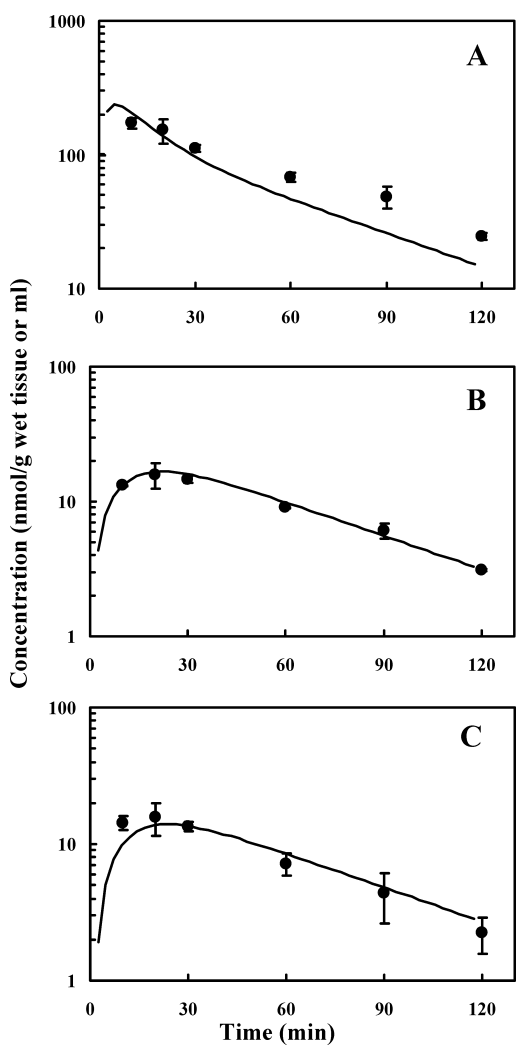

Fig. 2. Concentration of Timolol in the Cornea (A), Aqueous Humor (B), and Iris-Ciliary Body $(\mathrm{C})$ after Instillation

- Experimental data and (-) fitted curves. Each point represents the mean \pm S.E. of at least 3 experiments.

$\alpha+\beta=K_{\mathrm{TR} 1}+K_{\mathrm{R} 1 \mathrm{~T}}+K_{\mathrm{Te}}, \quad \alpha \cdot \beta=K_{\mathrm{R} 1 \mathrm{~T}} \cdot K_{\mathrm{Te}}$, and $(A \cdot \alpha+B \cdot \beta) /$ $(A+B)=K_{\mathrm{R} 1 \mathrm{~T}} . K_{\mathrm{TR} 1}$ and $K_{\mathrm{R} 1 \mathrm{~T}}$ are the transfer rate constants between the tear fluid and reservoir-1 (Res. 1) and $K_{\mathrm{Te}}$ is the elimination rate constant from the tear fluid. These parameters were estimated from the tear fluid concentrations after instillation of timolol using MULTI, a nonlinear leastsquares computer program. ${ }^{10}$

The ocular behaviors of timolol after instillation and injection into the anterior chamber (Figs. 2, 3) were analyzed by the PK model (Fig. 4), which was similar to our previous reported model for bunazosin. ${ }^{5)}$ Based on this model, the differential equations of drug amounts in the cornea $\left(X_{\mathrm{C}}\right)$, aqueous humor $\left(X_{\mathrm{A}}\right)$, iris-ciliary body $\left(X_{\mathrm{I}}\right)$, and Res. $2\left(X_{\mathrm{R} 2}\right)$ can be expressed as follows:

$$
\begin{aligned}
& \frac{d X_{\mathrm{C}}}{d t}=K_{\mathrm{TC}} \cdot X_{\mathrm{T}}+K_{\mathrm{AC}} \cdot X_{\mathrm{A}}-K_{\mathrm{CA}} \cdot X_{\mathrm{C}} \\
& \frac{d X_{\mathrm{A}}}{d t}=K_{\mathrm{CA}} \cdot X_{\mathrm{C}}+K_{\mathrm{R} 2 \mathrm{~A}} \cdot X_{\mathrm{R} 2}-\left(K_{\mathrm{AC}}+K_{\mathrm{AR} 2}+K_{\mathrm{AI}}+K_{\mathrm{Ae} 1}+K_{\mathrm{Ae} 2}\right) \cdot X_{\mathrm{A}}
\end{aligned}
$$$$
\frac{d X_{\mathrm{I}}}{d t}=K_{\mathrm{AI}} \cdot X_{\mathrm{A}}-K_{\mathrm{Ie}} \cdot X_{\mathrm{I}}
$$$$
\frac{d X_{\mathrm{R} 2}}{d t}=K_{\mathrm{AR} 2} \cdot X_{\mathrm{A}}-K_{\mathrm{R} 2 \mathrm{~A}} \cdot X_{\mathrm{R} 2}
$$

where $X_{\mathrm{T}}$ is the drug amount in the tear fluid, $K_{\mathrm{TC}}$ is the transfer rate constant from the tear fluid to the cornea, $K_{\mathrm{AI}}$ is the transfer rate constant from the aqueous humor to the irisciliary body, $K_{\mathrm{CA}}$ and $K_{\mathrm{AC}}$ are the transfer rate constants be-

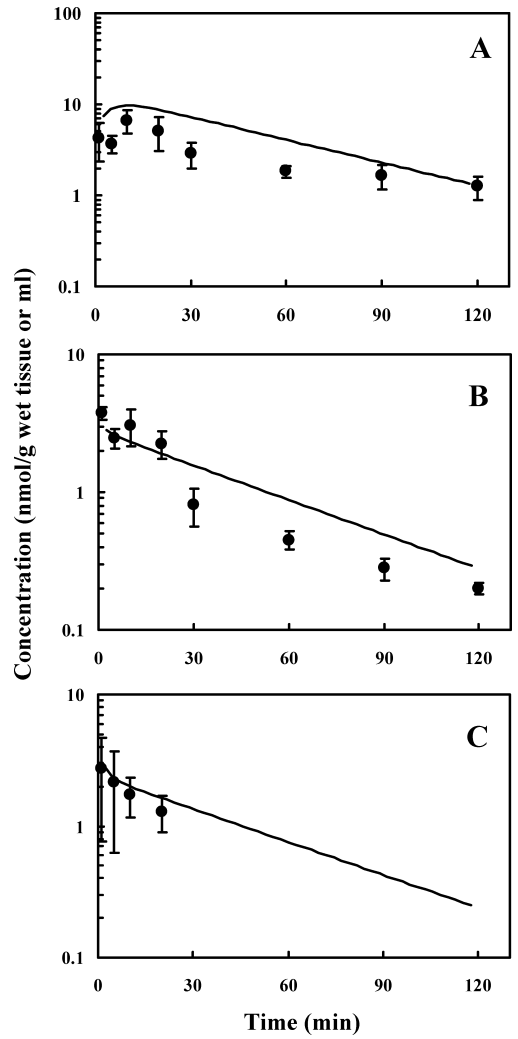

Fig. 3. Concentration of Timolol in the Cornea (A), Aqueous Humor (B), and Iris-Ciliary Body (C) after Ocular Injection into the Anterior Chamber

-) Experimental data and (-) fitted curves. Each point represents the mean \pm S.E. of at least 3 experiments.

tween the cornea and the aqueous humor, $K_{\mathrm{AR} 2}$ and $K_{\mathrm{R} 2 \mathrm{~A}}$ are the transfer rate constants between the aqueous humor and Res. $2, K_{\mathrm{Ie}}$ is the elimination rate constant from the irisciliary body, and $K_{\mathrm{Ae} 1}$ and $K_{\mathrm{Ae} 2}$ are elimination rate constants from the aqueous humor by aqueous humor flow and other routes. The value of $K_{\mathrm{Ae} 1}$ was obtained from the data reported by Sakurai M. et al. ${ }^{11)}$ The PK parameters were estimated from the drug amount-time profiles in the ocular tissues after instillation and injection. The estimations were carried out using MULTI (RUNGE), a nonlinear leastsquares computer program based on the Runge-Kutta-Gill method. ${ }^{12)}$

Ocular Pharmacokinetic/Pharmacodynamic Model for Timolol Based on the action mechanism of timolol, the ocular hypotensive effect-time profiles after instillation of timolol solutions (Figs. 5B to F) were analyzed by the following PK/PD model including aqueous humor dynamics (Fig. 6). An equilibrium of the aqueous humor inflow $\left(F_{\text {in }}\right)$ to the uveoscleral outflow $\left(F_{\text {us }}\right)$ and trabecular outflow $\left(F_{\text {tra }}\right) . F_{\text {tra }}$ is expressed by follows:

$$
F_{\text {tra }}=C_{\text {of }} \cdot\left(\mathrm{IOP}-P_{\mathrm{V}}\right)
$$

where $P_{\mathrm{V}}$ is the episcleral venous pressure and $C_{\mathrm{of}}$ is the outflow facility. Based on these relationships, IOP was expressed by aqueous humor flow as follows:

$$
\mathrm{IOP}=P_{\mathrm{V}}+\frac{F_{\text {in }}-F_{\text {us }}}{C_{\text {of }}}
$$

As timolol causes the reduction of IOP by suppressing aque- 

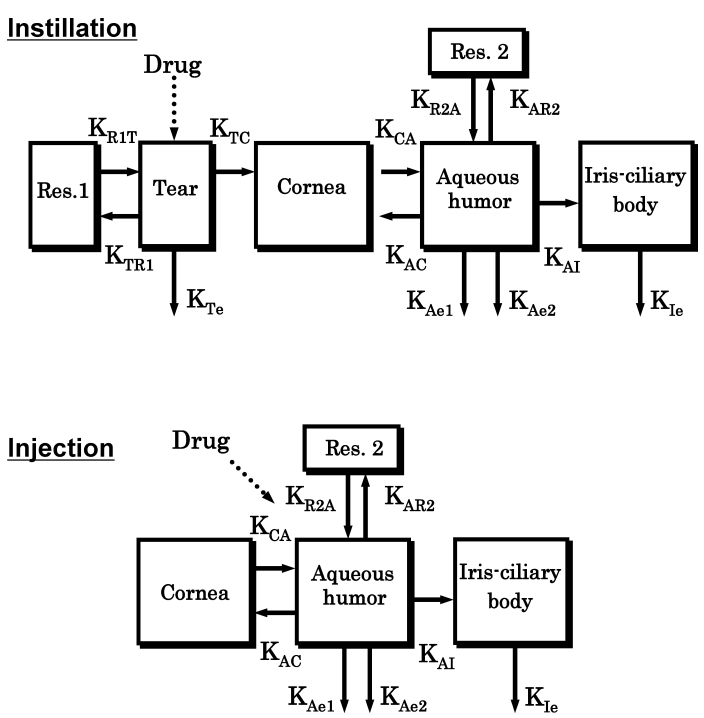

Fig. 4. Pharmacokinetic Models for Timolol in the Precorneal Area and Other Tissues after Instillation and after Ocular Injection into the Anterior Chamber
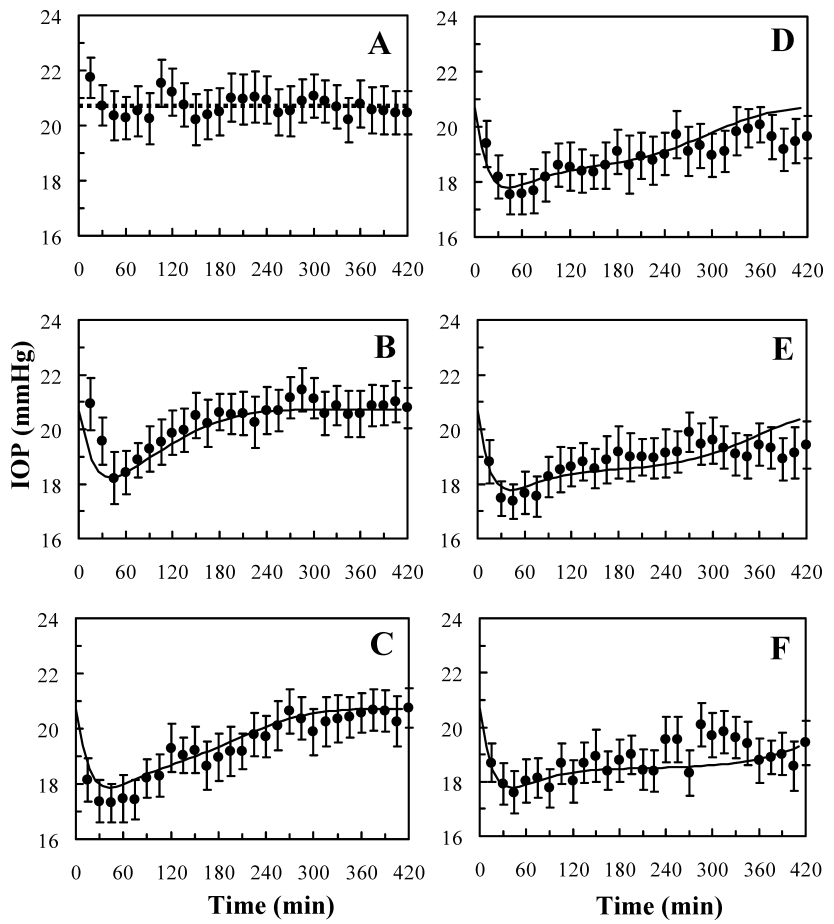

Fig. 5. Intraocular Pressures after Instillation of Vehicle or Timolol in Rabbits

(A) vehicle, (B) $0.2 \mathrm{~mm}$, (C) $1 \mathrm{~mm}$, (D) $5 \mathrm{~mm}$, (E) $15.8 \mathrm{~mm}$, and (F) $100 \mathrm{~mm}$. (-) Experimental data, (--) baseline, and (-) fitted curves. Each point represents the mean \pm S.E. of 16 experiments.

ous humor formation and does not change its outflow, ${ }^{13)}$ the differential equation of IOP after instillation of timolol can be expressed as follows:

$$
\frac{d \mathrm{IOP}}{d t}=\frac{d F_{\text {in }}}{d t} \cdot \frac{1}{C_{\text {of }}}
$$

An indirect response model ${ }^{14)}$ is applied for expressing the time delay in the maximum IOP reduction against aqueous humor concentration of timolol. This model represents a general approach where the rate of change in response is con-

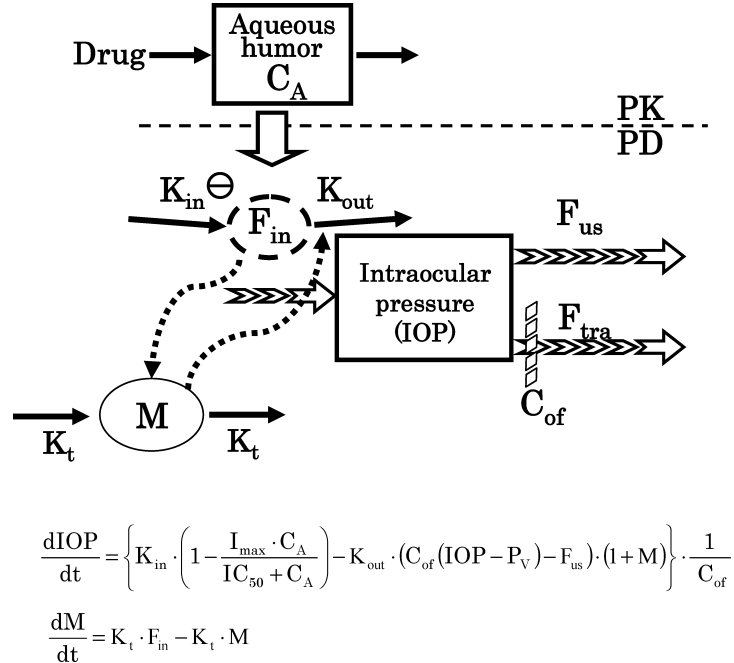

Fig. 6. Pharmacokinetic/Pharmacodynamic Model for Timolol

$\left(C_{\mathrm{A}}\right)$ drug concentration in the aqueous humor, (IOP) intraocular pressure, $\left(F_{\text {in }}\right)$ aqueous humor inflow, $\left(F_{\text {us }}\right)$ uveoscleral outflow, $\left(F_{\text {tra }}\right)$ trabecular outflow, $\left(C_{\text {of }}\right)$ outflow facility, $\left(P_{\mathrm{V}}\right)$ episcleral venous pressure, $\left(K_{\mathrm{in}}\right)$ zero-order rate constant, $\left(K_{\mathrm{out}}\right)$ first-order rate constant, $(M)$ regulator, $\left(K_{t}\right)$ first-order rate constant, $(I)$ maximum inhibitory effect, and $\left(\mathrm{IC}_{50}\right)$ the drug concentration that exhibits $50 \%$ of the maximum inhibitory effect.

trolled by a zero-order process (zero-order rate constant $K_{\text {in }}$ ) for production of the response and a first-order process (firstorder rate constant $K_{\text {out }}$ ) for loss of the response. In this model, $F_{\text {in }}$ production as the response is indirectly controlled by the drug concentration in the aqueous humor $\left(C_{\mathrm{A}}\right)$ and $F_{\text {in }}$ is lost at first-order. Furthermore, a slight overshoot phenomenon was recognized in ocular hypotensive responses. These responses can be explained by a push-and-pull model, ${ }^{15)}$ a kind of the indirect response model, for a drug acting as an inhibitor of production of response. This model has an additional compartment, which corresponds to a regulator, $M$. The push-and-pull model was also reported for describing tolerance and/or withdrawal syndrome against heart rate after systemic administration of propranolol. ${ }^{16)}$ Therefore, the differential equation of $F_{\text {in }}$ and $M$ can be expressed as follows:

$$
\begin{aligned}
& \frac{d F_{\text {in }}}{d t}=K_{\mathrm{in}}\left(1-\frac{I_{\text {max }} \cdot C_{\mathrm{A}}}{\mathrm{IC}_{50}+C_{\mathrm{A}}}\right)-K_{\mathrm{out}} \cdot F_{\mathrm{in}} \cdot(1+M) \\
& \frac{d M}{d t}=K_{\mathrm{t}} \cdot F_{\mathrm{in}}-K_{\mathrm{t}} \cdot M
\end{aligned}
$$

where $K_{\text {in }}$ is the zero-order rate constant for the production of $F_{\text {in }}$, and $K_{\text {out }}$ is the first-order rate constant for the loss of $F_{\text {in }}$. A decrease in $F_{\text {in }}$ causes a decrease in regulator governed by the first-order rate constant $\left(K_{\mathrm{t}}\right) . M$ is assumed to stimulate the loss of $F_{\text {in. }}$. It is assumed that $K_{\text {in }}$ and $K_{\text {out }}$ account for the production and loss of $F_{\text {in }} . I_{\max }$ is the maximum inhibitory effect attributed to the drug, and $\mathrm{IC}_{50}$ is the drug concentration that exhibits $50 \%$ of the maximum inhibitory effect.

When Eq. 7 and Eq. 9 were substituted in Eq. 8, we obtained the equation for the PK/PD model as follows:

$$
\begin{aligned}
\frac{d \mathrm{IOP}}{d t}= & \left\{K_{\mathrm{in}} \cdot\left(1-\frac{I_{\mathrm{max}} \cdot C_{\mathrm{A}}}{\mathrm{IC}_{50}+C_{\mathrm{A}}}\right)-K_{\mathrm{out}} \cdot\left(C_{\mathrm{of}}\left(\mathrm{IOP}-P_{\mathrm{V}}\right)-F_{\mathrm{us}}\right) \cdot(1+M)\right\} \\
& \times \frac{1}{C_{\mathrm{of}}}
\end{aligned}
$$

Physiological parameters, $P_{\mathrm{V}}, F_{\text {us }}$, and $C_{\text {of }}$, were obtained from the data reported by Sakurai et al. ${ }^{11)} C_{\mathrm{A}}$ is defined as 
$C_{\mathrm{A}}=X_{\mathrm{A}} / V_{\mathrm{A}}$. The aqueous humor volume, $V_{\mathrm{A}}$, is obtained from the data reported by Conrad and Robinson ${ }^{17)} \mathrm{PD}$ parameters, $K_{\mathrm{in}}, I_{\max }, \mathrm{IC}_{50}$, and $K_{\mathrm{t}}$ were estimated from the ocular hypotensive effect-time profiles after instillation of various concentration of timolol solutions. The estimations were carried out using MULTI (RUNGE). $K_{\text {out }}$ was estimated as $K_{\text {in }}$ and $F_{\text {in }}$ at zero time.

\section{RESULTS}

The concentration-time profiles of timolol in the ocular tissues were determined after its instillation into rabbit's eyes. Figure 1 shows the observed concentrations of timolol and its fitted curve in tear fluid. The observed profile showed a bi-exponential curve. The elimination rate constant and apparent distribution volume were estimated according to a two-compartment model. Figure 2 shows the observed concentrations of timolol and the fitted curves in the cornea, aqueous humor, and iris-ciliary body after instillation. The timolol concentrations in the cornea were much higher than those in the aqueous humor and the iris-ciliary body and reached a maximum at 10 min after dosing and then gradually decreased. The aqueous humor and the iris-ciliary body concentrations of timolol reached a maximum at 20 min after dosing and then gradually decreased. The concentration-time profiles of timolol in the cornea, aqueous humor, and iris-ciliary body were also determined after ocular injection into the anterior chamber of the eye of rabbits and are shown in Fig. 3 . The timolol concentrations in the cornea reached a maximum at 10 min after dosing and then gradually decreased. The aqueous humor and the iris-ciliary body concentrations of timolol continued to decrease after the injection. The timolol concentrations in the iris-ciliary body were below the lower limit of quantification $30 \mathrm{~min}$ after dosing.

The IOP of rabbits was determined continuously with a telemetry system. In a preliminary experiment, a typical circadian pattern was obtained. IOP increased in the dark phase, maintained a high, steady value until the end of the dark phase (steady phase 11:00 p.m. to 6:00 a.m.). Figure 5A shows the observed IOP-time profile after instillation of vehicle during the steady phase. The IOP value did not alter over the $7 \mathrm{~h}$ after vehicle instillation. The average IOP value after instillation of vehicle was $20.7 \mathrm{mmHg}$, and this was defined as the baseline value. Figs. 5B to $\mathrm{F}$ shows the ocular hypotensive effect-time profiles and their fitted curves after instillation of $0.2 \mathrm{~mm}, 1 \mathrm{~mm}, 5 \mathrm{~mm}, 15.8 \mathrm{~mm}$, and $100 \mathrm{~mm}$ timolol, respectively, in the steady phase. Instillation of timolol decreased the IOP value substantially. The time of maximum IOP reduction $(45 \mathrm{~min}$ ) was obviously later than that of the aqueous humor maximum concentrations. After instillation of $1 \mathrm{~mm}$ or higher concentrations of drug solution, all profiles were almost the same by $2 \mathrm{~h}$. In particular, a more emphasized reduction and a slight overshoot was observed at around $45 \mathrm{~min}$ after instillation. The IOP values returned to the baseline more gradually with escalating concentrations of drug solution.

\section{DISCUSSION}

Timolol, a representative $\beta$-adrenergic antagonist, is commercially available and used extensively as an anti-glaucoma agent. After topical application, timolol has been demonstrated to significantly lower IOP in rabbits ${ }^{3,8,13)}$ and humans. ${ }^{1)}$ Timolol penetrates mainly through the cornea, which acts as a depot for the drug ${ }^{18)}$ and permeates predominantly by passive diffusion. ${ }^{19)}$ To describe the ocular behaviors of timolol, some ocular PK models have already been reported as various compartment models. ${ }^{3,6)}$.In this study, the PK model as described in Fig. 4 was applied to a 6-compartment model. With regard to the theoretical concept, our PK model for timolol was compatible with previous reported findings.

In our previous study, the PK model for bunazosin described well the bunazosin concentrations in the tear fluid, aqueous humor, and other ocular tissues. ${ }^{5)}$ The PK model for timolol was developed by making appropriate modifications to the PK model for bunazosin in which the corneal compartment consisted of the corneal epithelium and its stroma. In the PK model for timolol, the corneal compartment was set as one compartment. It seems that the difference in corneal compositions depends on the physicochemical properties of the drugs. The PK parameters of timolol were estimated from two experiments using both instillation and injection into the anterior chamber of the eye to increase the reliability of PK parameters.

Estimated PK parameters are shown in Table 1. The fitting curves using the PK model and the PK parameters almost agreed with the observed concentrations (Figs. 1-3). It is also possible to incorporate the slight alteration of aqueous humor flow derived from the effect of timolol into this PK model by replacing $K_{\mathrm{Ael}}$, first-order constant, with an appropriate concentration-dependent parameter. In the present study, we used the above PK model consisting of 6 compartments to analyze the correlation of the ocular hypotensive effect-time profiles to the concentration-time profiles after instillation of timolol.

It is common using ocular hypertensive rabbits such as saline-loaded rabbits ${ }^{13)}$ or chymotrypsin-treated rabbits ${ }^{3)}$ to detect the IOP lowering effect of timolol, because the intensity of its ocular hypotensive effect in normotensive rabbits is relatively low and timolol reduces IOP only during the dark in normotensive rabbits. ${ }^{20)}$ To detect the IOP reduction of timolol using normotensive rabbits is difficult. Therefore, in order to develop the PK/PD model for timolol, we applied a telemetry system to measure the ocular hypotensive effect after its instillation in normotensive rabbits. This system

Table 1. Ocular Pharmacokinetic Parameters for Timolol

\begin{tabular}{lll}
\hline \hline Parameter & Value $\left(\mathrm{min}^{-1}\right)$ & \multicolumn{1}{c}{ S.D. } \\
\hline$K_{\mathrm{TR} 1}$ & 0.394 & 0.279 \\
$K_{\mathrm{R} 1 \mathrm{~T}}$ & 0.346 & 0.048 \\
$K_{\mathrm{Te}}$ & 0.271 & 0.033 \\
$K_{\mathrm{TC}}$ & $0.466 \times 10^{-3}$ & $0.036 \times 10^{-3}$ \\
$K_{\mathrm{CA}}$ & 0.195 & 0.028 \\
$K_{\mathrm{AC}}$ & 0.165 & 0.036 \\
$K_{\mathrm{AI}}$ & 0.107 & 0.146 \\
$K_{\mathrm{AR} 2}$ & 0.513 & 0.200 \\
$K_{\mathrm{R} 2 \mathrm{~A}}$ & 2.083 & 0.644 \\
$K_{\mathrm{Ae} 1}$ & $0.010^{a)}$ & - \\
$K_{\mathrm{Ae} 2}$ & $1.66 \times 10^{-3}$ & $1.46 \times 10^{-3}$ \\
$K_{\mathrm{Ie}}$ & 0.750 & 1.053 \\
\hline
\end{tabular}

a) Value was reference from reported data. ${ }^{11)}$ 
Table 2. Ocular Pharmacodynamic Parameters for Timolol

\begin{tabular}{lll}
\hline \multicolumn{1}{c}{ Parameter } & \multicolumn{1}{c}{ Value } & \multicolumn{1}{c}{ S.D. } \\
\hline$K_{\text {in }}\left(\mu \mathrm{l} / \mathrm{min} \cdot \min ^{-1}\right)$ & 0.148 & 0.038 \\
$K_{\text {out }}\left(\mathrm{min}^{-1}\right)$ & $0.0663^{a)}$ & - \\
$I_{\text {max }}$ & 0.268 & 0.017 \\
$\mathrm{IC}_{50}(\mathrm{nmol} / \mathrm{ml})$ & $5.71 \times 10^{-3}$ & $1.46 \times 10^{-3}$ \\
$K_{\mathrm{t}}\left(\mathrm{min}^{-1}\right)$ & 0.0152 & 0.0068 \\
$F_{\text {us }}(\mu \mathrm{l} / \mathrm{min})$ & $0.25^{b)}$ & - \\
$C_{\text {of }}(\mu \mathrm{l} / \mathrm{min} / \mathrm{mmHg})$ & $0.170^{b)}$ & - \\
$P_{\mathrm{V}}(\mathrm{mmHg})$ & $9.00^{b)}$ & - \\
\hline
\end{tabular}

a) $K_{\text {out }}$ was estimated as $K_{\text {in }}$ and $F_{\text {in }}$ at zero time. b) Values were reference from reported data. ${ }^{11)}$

could produce IOP data applicable for PK/PD analysis every 15 min automatically after instillation without any handling. Akaishi et al. reported that the circadian variation was measurable and IOP reduction by timolol was detectable only in the dark when using the telemetry system. ${ }^{8)}$ In the present study, obvious IOP reductions caused by timolol instillation were obtained by the telemetry system. These results were in agreement with the previous report.

According to the analysis of the ocular hypotensive effect-time profiles after instillation of various concentrations of timolol solution, it was clear that the profiles showed two characteristic features. One was the time delay in the maximum IOP reduction against the aqueous humor maximum concentration, and the other was the slight overshoot phenomenon around the maximum effect time. In particular, the slight overshoot phenomenon could only be detected by detailed observations with the telemetry system. Timolol, which has also been reported as an inverse agonist, ${ }^{21)}$ may cause the overshoot phenomenon due to the rapid exposure to enormous level of the drug because the inverse agonism stabilizes $\beta$-receptors into their inactive state. ${ }^{22)}$ Based on the above results, the PK/PD model, as described in Fig. 6, was developed.

The PK/PD model described well the ocular hypotensive effect-time profiles after the instillation of timolol. Estimated PD parameters are shown in Table 2. The estimated $\mathrm{IC}_{50}$ value $(5.71 \mathrm{~nm})$ of timolol almost agreed with the reported receptor binding inhibitory constant $\left(K_{\mathrm{i}}\right)$ values for timolol $\left(K_{\mathrm{i}}=1.97 \mathrm{~nm}\right.$ for $\beta_{1}$ adrenergic receptors, $K_{\mathrm{i}}=2.00 \mathrm{~nm}$ for $\beta_{2}$ adrenergic receptors ${ }^{23)}$ ). The estimated $I_{\max }$ value for timolol $(0.268)$ shows that the $\beta$-adrenergic receptor mediated inhibitory effect of timolol does not completely suppress $K_{\text {in }}$, which is associated with aqueous humor production. This finding agreed with the previous report that timolol significantly reduced $(40 \%)$ the rate of aqueous humor formation after instillation of a $1 \%$ timolol solution in hypertonic saline-loaded rabbits. ${ }^{13)}$ By regarding the $\mathrm{IC}_{50}$ as dissociation constant, $K_{\mathrm{d}}$, upon $\beta$-receptor, the receptor occupancies of timolol $1 \mathrm{~h}$ after instillation of $0.2 \mathrm{~mm}, 1 \mathrm{~mm}, 5 \mathrm{~mm}, 15.8 \mathrm{~mm}$, and $100 \mathrm{~mm}$ were $77.5 \%, 94.5 \%, 98.9 \%, 99.6 \%$, and $99.9 \%$, respectively. A receptor occupancy model can be produced from this PK/PD model. This model may be applicable for similar analysis of other $\beta$-adrenergic antagonists, because the model includes aqueous humor dynamics based on an action mechanism of $\beta$-adrenergic antagonist, timolol.

\section{CONCLUSIONS}

We developed an ocular PK/PD model including dynamics of the aqueous humor based on the action mechanism of timolol. This model described well both the concentration of timolol in the ocular tissues and the ocular hypotensive effects after instillation of timolol in rabbits. This model will be effective to estimate the adequate regimens for ophthalmic pharmacotherapy and to develop improved ocular drug delivery systems for glaucoma. The telemetry system, which was able to automatically obtain detailed ocular hypotensive effect-time profiles in rabbits, was applicable to complex $\mathrm{PK} / \mathrm{PD}$ analysis. This study is the first trial to develop an ocular PK/PD model for timolol after instillation.

Acknowledgments The author wishes to thank Dr. Yamaoka for supplying MULTI (RUNGE).

\section{REFERENCES}

1) Woodward D. F., Chen J., Expert Opin. Emerg. Drugs, 12, 313-327 (2007).

2) Zhan G.-L., Toris C. B., Camras C. B., Wang Y.-L., Yablonski M. E., J. Ocul. Pharmacol. Ther., 14, 217-228 (1998).

3) Chiang C.-H., Ho J.-I., Chen J.-L., J. Ocul. Pharmacol. Ther, 12, $471-480$ (1996).

4) Zimmer A., Mutschler E., Lambrecht G., Mayer D., Kreuter J., Pharm. Res., 11, 1435-1442 (1994).

5) Sakanaka K., Kawazu K., Tomonari M., Kitahara T., Nakashima M., Kawakami S., Nishida K., Nakamura J., Sasaki H., Pharm. Res., 21, $770-776$ (2004).

6) Francoeur M. L., Sitek S. J., Costello B., Patton T. F., Int. J. Pharm., 25, 275-292 (1985).

7) Sasaki H., Ichikawa M., Kawakami S., Yamamura K., Mukai T., Nishida K., Nakamura J., J. Pharm. Pharmacol., 49, 140-144 (1997).

8) Akaishi T., Ishida N., Shimazaki A., Hara H., Kuwayama Y., J. Ocul. Pharmacol. Ther, 21, 436- 444 (2005).

9) McLaren J. W., Brubaker R. F., FitzSimon J. S., Invest. Ophthalmol. Vis. Sci., 37, 966-975 (1996).

10) Yamaoka K., Tanigawara Y., Nakagawa T., Uno T., J. PharmacobioDyn., 4, 879-885 (1981).

11) Sakurai M., Araie M., Oshika T., Mori M., Shoji N., Masuda K., Jpn. J. Ophthalmol., 37, 252-258 (1993).

12) Yamaoka K., Nakagawa. T., J. Pharmacobio-Dyn., 6, 595-606 (1983).

13) Vareilles P., Lotti V. J., Ophthalmic Res., 13, 72 -79 (1981).

14) Gabrielsson J., Weiner D., "Pharmacokinetic and Pharmacodynamic Data Analysis: Concepts and Applications," 2nd ed., Swedish Pharmaceutical Press, Stockholm, 1997, pp. 625-632.

15) Gabrielsson J., Weiner D., "Pharmacokinetic and Pharmacodynamic Data Analysis: Concepts and Applications," 2nd ed., Swedish Pharmaceutical Press, Stockholm, 1997, pp. 646-660.

16) Paalzow L. K., Själander-Brynne L., Adv. Drug Deliv. Rev., 33, 235240 (1998).

17) Conrad J. M., Robinson J. R., J. Pharm. Sci., 66, 219-224 (1977).

18) Araie M., Takase M., Yokoyama Y., Kitagawa M., J. Jpn. Ophthalmol. Soc., 84, 2139-2146 (1980).

19) Sakanaka K., Kawazu K., Nishida K., Nakamura J., Nakashima M., Nakamura T., Oshita A., Ichikawa N., Sasaki H., Biol. Pharm. Bull., 29, 2143-2147 (2006).

20) Gregory D. S., Invest. Ophthalmol. Vis. Sci., 31, 715-721 (1990).

21) Chidiac P., Hebert T. E., Valiquette M., Dennis M., Bouvier M., Mol. Pharmacol., 45, 490-499 (1994).

22) Metra M., Cas L. D., Cleland J. G. F., J. Card. Fail., 12, 177-181 (2006).

23) Phan T.-M. M., Nguyen K. P. V., Giacomini J. C., Lee D. A., J. Ocul. Pharmacol., 7, 243-252 (1991). 